\title{
How do Continuous Climb Operations affect the capacity of a Terminal Manoeuvre Area?
}

\author{
Javier A. Pérez-Castán, Fernando Gómez Comendador, Rosa M. Arnaldo Valdés \\ School of Aeronautical Engineering (ETSIAE), Technical University of Madrid (UPM) \\ javier.perez.castan@upm.es, fernando.gcomendador@upm.es and \\ rosamaria.arnaldo@upm.es
}

\begin{abstract}
Continuous climb operations are the following step to optimise departure trajectories with the goals of minimizing fuel consumption and pollutants and noise emissions in the airports neighbourhood, although due to intrinsic nature of these procedures, the integration of these procedures need to develop a new framework for airline operators and air traffic control. Based on the BADA model developed by EUROCONTROL, three activities have been carried out: simulation of several continuous climbs for three aircraft types (Light, Medium and Heavy), analysation of different applied separations throughout the climb from the runway up to cruise level and, as third activity, definition of new separation minima to ensure that the minimum separations are not violated with this new procedures along the climb. In this work are presented the results of modelling three continuous climb type (constant true airspeed, constant climb angle and constant vertical speed) and new time-based separations for most used models in Palma TMA, which will be the case-study scenario. Finally, this theoretical analysis has been applied to a real scenario in Palma de Mallorca TMA in order to compare how the capacity deals with the introduction of this new procedure to standard departures, standard departures are understood as a departure with a level-off at a determined altitude and with the possibility to be affected by any ATC action. First outcomes are promising because capacity, theoretically, would not be grossly diminished, which could initially be expected based on previous studies on continuous descent approaches, although these results should be considered cautiously due to the fact that the model lacks several factors of associated uncertainty for a real climb.
\end{abstract}

\section{INTRODUCTION}

Air Transport is one of the largest worldwide industries, according to (OACI, 2013), nearly of 3100 million of passengers took the air transport network in 2013. The number of passengers increased over 5\% compared with 2012 and it is expected to reach more than 6400 million to 2030 . This increase of demand prompts the necessity to perform a future restructuration of the worldwide airspace (SESAR, 2014), by the implementation of novel air procedures which will allow aircrafts to guide themselves over more accuracies 
trajectories, decreasing the time of flight and likewise helping to reduce the environmental impact.

On the last decade significant research has been carried out over climb operations by major stakeholders as NASA (ROACH, 2010), FAA, EUROCONTROL, etc. The Continuous Climb Operation (CCO) aim is to enable aircraft to fly their optimal path within the requirements of the ATC system. IFATCA (2012) presented a literature review, up to 2012, and analysed the requirements that a CCO should fulfil if a new procedure is defined. The potential problems outlined were that these new procedures are more complex and, therefore, will increase operational risk and the compliance with the vertical profile would be more difficult to monitor and distinguish which aircraft is flying a CCO or a level-off departure.

CCO modelling is based on Continuous Descent Approach (CDA) developments because the CDA modelling was built before (TONG, 2007). The theoretical integration of these procedures (MELBY, 2008; MILLER, 2011) concludes that remarkable USD savings can be achieved with this implementation. Moreover, the potential benefits in the environmental frame can be very high in terms of pollutants and noise emissions. Thus, for all these reasons, an important number of research have analysed the CCO potential to reduce these environmental aspects with different optimization approaches (KHARDI, 2010; PRATS, 2010; TORRES, 2011).

Based on studies performed on the integration of CDAs in a Terminal Manoeuver Area (TMA), it is known that the implementation of these procedures suppose a significant capacity reduction (ALAM, 2010; REN, 2003; WEITZ, 2005), because releasing the descent operation of possible ATC actions, as level-offs or speed changes, supposes that the separation minima must be enlarged to assure that will not be violated. However, the inverse analysis of how the integration of CCOs affect the capacity of a TMA has not been tackled so far in-depth.

This work is organized as follows: A conceptual framework for continuous climb procedures is presented in the next section with the development of the BADA model and some results of the benefits of a CCO. This is followed by a discussion of the relationship between TMA and airport operational capacity based on Palma TMA (SPAIN) scenario and the viability of CCO integration. The major findings of this research and the outlines of future works are summarized in the last section.

\section{CONTINUOUS CLIMB OPERATION}

\subsection{Continuous Climb Operation concept}

A CCO is an operational procedure characterized by the execution of a climb according just 
to the aircraft performance without ATC restrictions: level-offs, headings or speed changes are eliminated in order to let a 'free' trajectory throughout its climb. By this way, the CCO aircraft climbs with its optimal profile in which it can optimize several factors. Currently the main factors to reduce in airports neighbourhoods are the fuel consumption and the pollutants and noise emissions which suppose restrictions for the airport capacity nowadays and hardly in the future. Indirect factors that will be improved with the use of CCO are the time and distance to reach the cruise level. This potential savings and the following drawbacks for the air traffic management agree with the analysis carried out by Reynolds (2014):

- Major uncertainty because each aircraft has a different optimal climb profile.

- Need to increase distance between following aircrafts to ensure that at any time minimum safety requirements are violated.

- Need to increase time windows into potential conflict points among departure and landing flows.

- Lastly, an operational capacity reduction which could inhibit the integration of CCO with step-traffic.

\subsection{Continuous Climb Operation model}

In this section the modelling of distinct stages of a $\mathrm{CCO}$ procedure is described and the simulation of three CCO types are presented. The CCO structure is bounded by the restrictions applied to the aircraft operational characteristics along the take-off and climb phase and with the vanishing of any ATC or ATM bound. So that three stages are distinguished:

1. From take-off up to $1.500 \mathrm{ft}$.: this section is defined by climbing in take-off configuration with maximum thrust and extended flaps.

2. From $1.500 \mathrm{ft}$. up to transition altitude $H_{p}$ : the aircraft climbs according to its optimal profile, clean (cruise) configuration and the ATC restriction of $v_{\max }=250$ IAS up to FL100 disappears.

3. From $H_{p}$ up to cruise level: the aircraft changes the speed operational concept to Mach operational concept, where Mach $=v_{t a s} / a$ and $a$ is the sound speed.

In this research three different continuous climb models have been put into practice based on different operational concept:

- Constant true airspeed $v_{\text {tas }}=c t e$;

- Constant climb angle $\gamma=c t e$, and,

- Constant vertical speed $\dot{h}=$ cte.

These models have been implemented to distinct aircraft types following the aircraft model of BADA (EUROCONTROL, 2014). In Table 1 is presented, for a medium type aircraft (B737), the summary of the results for three key factors (fuel consumption, time and 
horizontal distance) in order to assess the benefits of each CCO. Besides, this results are compared with a 'standard' departure which climbs with $v_{\text {tas }}=$ cte concept and performs a level-off of 3 min at FL90.

\begin{tabular}{|c|c|c|c|}
\hline \multirow{2}{*}{ Operational concept } & \multicolumn{3}{|c|}{ Key Factors } \\
\cline { 2 - 4 } & Fuel consumption $(\mathrm{kg})$ & Time $(\mathrm{s})$ & Horizontal distance $(\mathrm{km})$ \\
\hline$v_{\text {tas }}=350 \mathrm{kts}$ & 1261.5 & 865 & 155.8 \\
\hline$\gamma=2.5^{\mathrm{o}}$ & 1766.9 & 1015 & 230.1 \\
\hline$\dot{h}=2000 \mathrm{ft} / \mathrm{min}$ & 1894.3 & 1098 & 255.2 \\
\hline 'Standard' departure & 1661.5 & 1211 & 169.2 \\
\hline
\end{tabular}

Table 1 - Results in terms of key factors of CCO simulation with the BADA model depending on the operational concept.

Firstly, because space reasons solely best cases for each CCO type has been presented in Table 1, for each operational concept considered variations have been assessed in order to appreciate how these variations affect the climb profile: from 150 to $400 \mathrm{kts}$ variations for $v_{\text {tas }}=$ cte ; from 1 to $5^{\circ}$ for $\gamma=c t e$ and from 500 to $2500 \mathrm{ft} / \mathrm{min}$ for $\dot{h}=$ cte.. As it is observed in Table 1, best outputs for fuel consumption, time and distance are for $v_{\text {tas }}=$ cte and it is clear the influence of the level-off in terms of fuel consumption and time. In Figure 1, the corresponding CCO profiles of the best cases are represented:
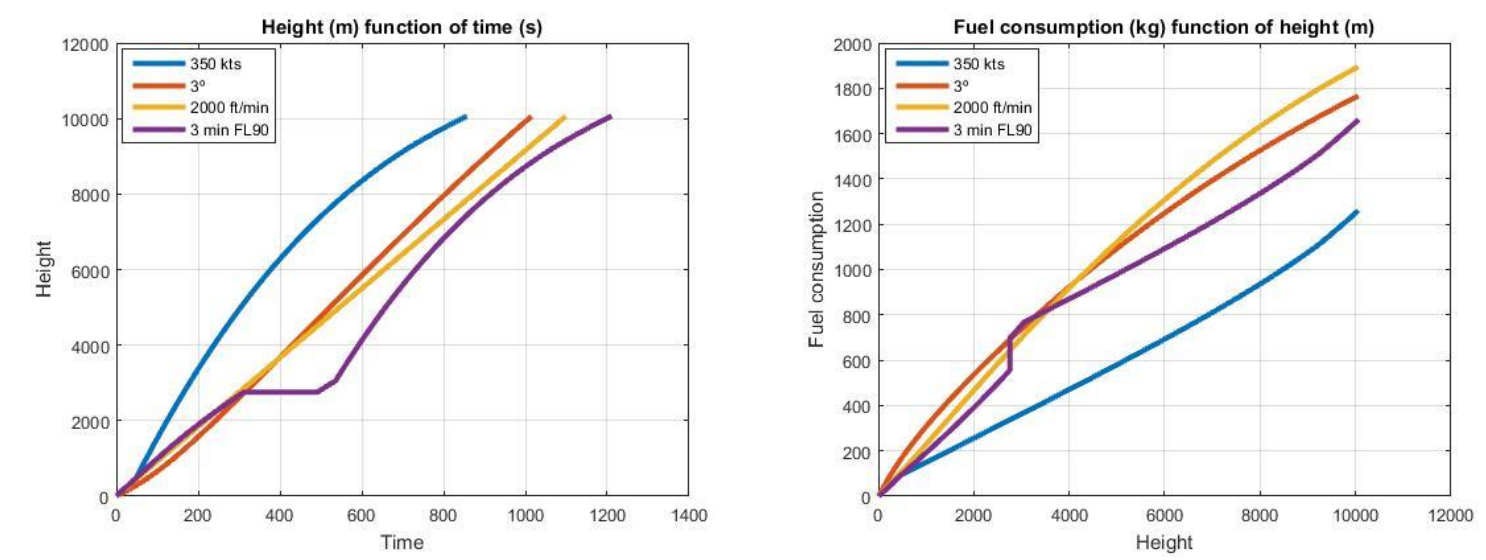

Figure 1 - Trajectory of the three CCO types: a) Height function of time and b) Fuel consumption function of height.

\section{TMA - RUNWAY SEPARATION: CASE-STUDY PALMA}

The aim of this module is to analyse how affects the separations between aircrafts the integration of CCO procedures in the different parts of a TMA in order to assure the safety of the departures and the separation minima. TMA capacity is constrained by diverse factors as traffic density, arrival and departure trade-offs, airspace design, SID and STAR procedures, number of airports inside TMA, potential conflict points, etc. Thus, in order to 
consider the integration of these procedures into a high-density real scenario, Palma TMA traffic has been deeply analysed with the NEST tool (EUROCONTROL, 2013).

In this research, as a first approach of separation assessment, separation between aircraft types are defined as a measure of the TMA - Runway capacity. Firstly, depending on the spatial location of the aircrafts they are distinguished three types of separations which must be applied for the departure traffic: runway, TMA and en-route. The definition of each one depends on the following factors:

- Standard Instrumental Departure (SID) allocation,

- Aircraft pair type, wake-turbulence minima, and

- Ground-navigation systems, radar coverage or not.

In the case of the leading aircraft SID differs from the trailing aircraft just after taking-off, the Spanish RCA (MINISTERIO DE FOMENTO, 2014) specifies that the separation minima must be of $1 \mathrm{~min}$, whereas aircrafts using the same SID, or partially the same, the separation must be of $2 \mathrm{~min}$. In the case of wake-turbulence minima the following minima must be applied depending on the aircraft pair and the separation type considered:

\begin{tabular}{|c|c|c|c|}
\hline Leading aircraft & Trailing aircraft & Time & Distance \\
\hline Heavy & Heavy & 2 min & $4 \mathrm{NM}$ \\
\hline Heavy & Medium & $2 \mathrm{~min}$ & $5 \mathrm{NM}$ \\
\hline Heavy & Light & $2 \mathrm{~min}$ & $6 \mathrm{NM}$ \\
\hline Medium & Light & $2 \mathrm{~min}$ & $5 \mathrm{NM}$ \\
\hline
\end{tabular}

Table 2 - Wake-turbulence separation minima.

TMA separation minima depends on the existence of radar coverage or not and if it does exist is of 3 or $5 \mathrm{NM}$ depending on the distance between aircraft and airport, in Palma TMA is $3 \mathrm{NM}$. On the other hand, en-route separation minima with radar coverage are $5 \mathrm{NM}$.

Currently, ATC can act to assure the minima separation between departure aircrafts, but with the integration of CCOs they have to let freely the flight up to reach the cruise level, in other words, ATC cannot indicate the CCO aircraft to perform a level-off or vectoring or any speed change. The fundamental change of this procedure is that the trailing aircraft cannot take-off unless it does exist the certainty that it is not going to violate the separation with the precedent aircraft along the climb. That means that new separations have to be calculated/defined to assure this concept.

In order to quantify the new shape of these minima several simulations among three types of aircraft have been tested, an example of a CCO pair is presented in Figure 2. The separation is time-based and they have been calculated based on the time spend to reach the wake-turbulence minima in Nautical Miles. Palma TMA scenario is featured by a major aircraft for each aircraft type: PRM1 (Light), B737 (Medium) and A332 (Heavy), which 
have been used to simulate $\mathrm{CCO}$ and standard departure.
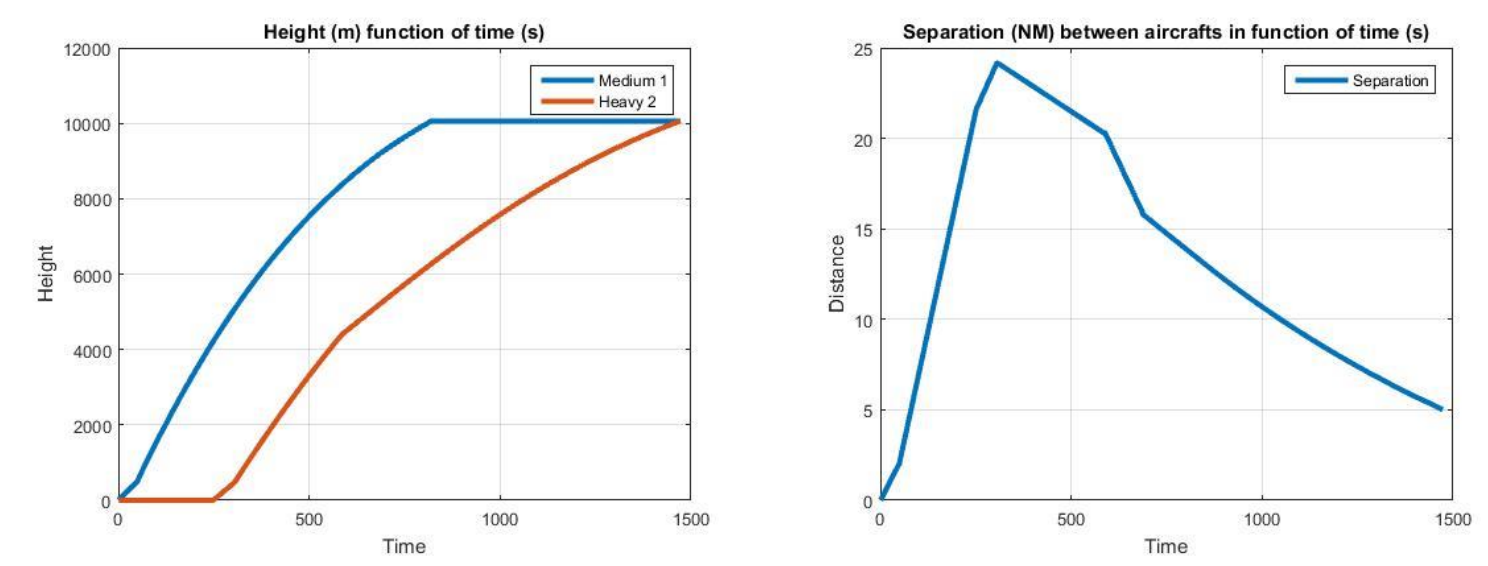

Figure 2 - Trajectories of Medium - Heavy pair performing CCOs: a) Height function of time and b) Separation between aircrafts.

Standard departure pairs have been simulated and tested in order to quantify the separation necessary to ensure the safety of the operations. As they have been defined in section 2 , the results in the Figure 3 are calculated to permit a standard departure any ATC, namely, the aircraft pair must be separated by the separation minima based on distance procedure of Table 2. Both CCO and standard results are showed in Table 3:

\begin{tabular}{|c|c|c|c|c|c|c|}
\hline & \multicolumn{3}{|c|}{ CCO } & \multicolumn{3}{c|}{ Standard Departure } \\
\hline Aircraft type & Light & Medium & Heavy & Light & Medium & Heavy \\
\hline Light & 61 & 113 & 436 & 61 & 61 & 61 \\
\hline Medium & 71 & 60 & 250 & 71 & 60 & 60 \\
\hline Heavy & 86 & 77 & 68 & 86 & 77 & 68 \\
\hline
\end{tabular}

Table 3 - CCO and Standard Departure runway separation minima in seconds.

The first conclusion is that $\mathrm{CCO}$ separations are better in various cases than 2 minute waketurbulence minima so, initially, they will enhance the time-based capacity. Compared with standard departure separations, there are the same separations except three cases (L-M or H and $\mathrm{M}-\mathrm{H}$ ) because in these cases the trailing aircraft is bigger than the precedent so the leading aircraft will be reached by the bigger one, therefore, the CCO separation must be increased. On the other hand, these pair, for standard departures cases, will suppose the interaction and merging of ATC actions and that will reduce as well the capacity.

Palma capacity in rush hour is 34 departures, so, theoretically, the integration of CCO would not suppose a great impact because the $95 \%$ of the aircrafts are Medium and the M-M separation is of $60 \mathrm{sec}$. Although first results are promising, this is a first approach in which several factors have not been considered, i.e., speed variations in climb profile, pilot errors, temperature influence, wind and so on, that will create uncertainties which will enlarge these 
initial separations and maybe will forbid the use of CCO in rush hours.

\section{CONCLUSIONS AND FUTURE WORK}

Nowadays air transport focuses on the development of more efficient and optimal procedures with the goal of reduce fuel consumption and pollutants and noise emissions albeit rarely it is analysed the influence that these changes produce into the airspace capacity. This research is focalised on the assessment of the integration of CCOs in a real scenario, Palma TMA, for that it has been performed a methodology based on BADA model for the simulation of CCO trajectories depending on three operational concepts: $v_{\text {tas }}, \dot{h} y \gamma$ constant. The results produced in terms of fuel consumption, time and distance confirm that $v_{\text {tas }}=$ cte climb provides best results. Once the $\mathrm{CCO}$ benefits are known, it has been considered how affects the execution of CCOs into the separation minima inside a TMA scenario, concerning mainly to the runway separation as a function of aircraft type and the connection with standard (level-off) departure separation. The separation minima has been calculated as well for the three major aircraft models that operate in Palma de Mallorca (PRM1, B737 and A332). Lastly, the implementation of these calculated minima have been assessed in a real and high-density scenario, Palma TMA and airport, obtaining a first positive outcome because the capacity is not reduced. Although, this is a theoretical result that has not considered uncertainties associated with any real climb as wind, pilot or on-board systems errors, etc., which will suppose an increase on the separation minima. Therefore, future work to develop among other research lines are: to add diverse aircraft models, to introduce several influence factors that will directly affect to the climb profile, to analyse the interaction of $\mathrm{CCO}$ with arrival flows, to model separations between $\mathrm{CCO}$ and standard departures mixed and to model the collision risk of these new procedures.

\section{ACKNOWLEDGEMENTS}

This Project has been developed under the programe "Plan estatal de Innovación Científica y Técnica y de Innovación 2013-201, Programa Estatal de Investigación, Desarrollo e Innovación Orientada a los Retos de la Sociedad: Development and optimization of takeoff and climb procedures for a smarter, sustainable and integrated air transport air transport system" with the collaboration of the Rey Juan Carlos University.

\section{REFERENCES}

ALAM, S., et al. (2010). A dynamic continuous descent approach methodology for low noise and emission. Digital Avionics Systems Conference (DASC), 2010 IEEE/AIAA 29th. IEEE, 2010. p. 1. E. 5-1-1. E. 5-18.

EUROCONTROL. (2013). NEST: Network Strategic Tool. 
http://www.eurocontrol.int/services/nest-modelling-tool.

EUROCONTROL. (2014). User Manual for the Base of Aircraft Data (BADA) Revision 3.12. EEC Technical/Scientific Report No. 14/04/24-44.

IFATCA. (2012). Study Continuous Climb Operations. 51 $1^{\text {st }}$ Annual Conference Kathmandu, $12^{\text {th }}$ to $16^{\text {th }}$ March 2012, WP No.85.

KHARDI, Salah. (2010). Mathematical model for advanced CDA and take-off procedures minimizing aircraft environmental impact. International mathematical Forum. 2010. p. 1747-1774.

MELBY, Paul; MAYER, Ralf H. (2008). Benefit potential of continuous climb and descent operations. The 26th Congress of International Council of Aeronautical Sciences (ICAS). 2008. p. 14-19.

MILLER, Mary Ellen; GRAHAM, Michael; ALDOUS, James. (2011). Efficient climb and descent benefit pool. Digital Avionics Systems Conference (DASC), 2011 IEEE/AIAA 30th. IEEE, 2011. p. 2E1-1-2E1-11.

MINISTERIO DE FOMENTO. (2014). Reglamento del aire y disposiciones operativas comunes para los servicios y procedimientos de navegación aérea. BOE-A-2014-6856. p. 50141-50319.

OACI. (2013). Air Traffic Press Release. http://www.icao.int/Newsroom/Pages/ICAOpredicts-continued-traffic-growth-through-2015.aspx.

PRATS, Xavier, et al. (2010). Multi-objective optimisation for aircraft departure trajectories minimising noise annoyance. Transportation Research Part C: Emerging Technologies, 2010, vol. 18, no 6, p. 975-989.

REN, Liling; CLARKE, John-Paul; HO, Nhut Tan. (2003). Achieving low approach noise without sacrificing capacity. Digital Avionics Systems Conference, 2003. DASC'03. The 22nd. IEEE, 2003. p. 1. E. 3-1.1-9 vol. 1.

REYNOLDS, Tom G. (2014). Air traffic management performance assessment using flight inefficiency metrics. Transport Policy, 2014, vol. 34, p. 63-74.

ROACH, Keenan; ROBINSON, John. (2010). A terminal area analysis of continuous ascent departure fuel use at Dallas/fort worth international airport. AIAA Aviation Technology, Integration and Operations Conference (ATIO). 2010.

SESAR. (2014). Modernising the European Sky. http://www.sesarju.eu/sites/default/files/documents/reports/brochure-2014-modernisingeuropean-sky-final.pdf.

TONG, Kwok-On, et al. (2007). Descent profile options for continuous descent arrival procedures within 3d path concept. Digital Avionics Systems Conference, 2007. DASC'07. IEEE/AIAA 26th. IEEE, 2007. p. 3. A. 3-1-3. A. 3-11.

TORRES, R., et al. (2011). Optimal, environmentally friendly departure procedures for civil aircraft. Journal of Aircraft, 2011, vol. 48, no 1, p. 11-22.

WEITZ, Lesley A., et al. (2005).An analysis of merging and spacing operations with continuous descent approaches. Digital Avionics Systems Conference, 2005. DASC 2005.

The 24th. IEEE, 2005. p. 2.3. C-21-11 Vol. 1. 